\title{
CORRELAÇÃO DO SINAL DE LASĖGUE E MANOBRA DA ELEVAÇÃO DA PERNA, RETIFICADA COM OS ACHADOS CIRÚRGICOS EM PACIENTES COM CIATALGIA PORTADORES DE HÉRNIA DISCAL LOMBAR
}

\author{
CORRELATION OF LASĖGUE SIGN AND THE STRAIGHT-LEG-RAISING TEST WITH SURGICAL \\ FINDINGS IN PATIENTS WITH SCIATICA AND LUMBAR DISC HERNIATION
}

\author{
CORRELACIÓN DEL SIGNO DE LASĖGUE Y MANIOBRA DE ELEVACIÓN DE LA PIERNA \\ RECTA CON LOS RESULTADOS QUIRÚRGICOS EN PACIENTES CON \\ CIÁTICA QUE TENIAN HERNIA LUMBAR DISCAL
}

Jonatas Sanchez Fernandez ${ }^{1}$, Afrane Serdeira², Marcus Sofia Ziegler³ ${ }^{3}$ Carlos Marcelo Donazar Severo ${ }^{4}$, Erasmo de Abreu Zardo ${ }^{5}$

\begin{abstract}
RESUMO
Objetivo: Investigar a correlação entre as manobras de estiramento do nervo ciático, como o Teste de Lasègue (TL) e o Teste de Elevação da Perna Estendida (TEPE), com os achados cirúrgicos nos pacientes com ciatalgia. Métodos: 178 pacientes portadores de hérnia de disco lombar foram examinados previamente pelo autor através do TL e do TEPE. Os achados cirúrgicos foram anotados e comparados com os achados do exame clínico. Resultados: Dos pacientes estudados, 162 (91\%) apresentaram TL positivo enquanto 118 (66,2\%) apresentaram a TEPE positivo. Conclusão: O TL foi a manobra diagnóstica pré-operatória mais sensível em correlação com a hérnia discal lombar.
\end{abstract}

Descritores: Deslocamento do disco intervertebral; Discotomia; Vértebras lombares; Exame físico; Doenças da coluna vertebral.

\begin{abstract}
Objective: To investigate the correlation between maneuversfor stretching the sciatic nerve, as the Lasègue Sign (LS) and the Straight-Leg-Raising Test (SLRT), with surgical findings in patients with sciatica. Methods: 178 patients with herniated lumbar disc were previously examined by the author through LS and SLRT. Surgical findings were recorded and compared with findings on clinical examination. Results: Of the patients studied, 162 (91\%) had positive LS while 118 (66.2\%) were positive to SLRT. Conclusion: The LS was the most sensitive preoperative physical diagnostic test with respect to intraoperative pathology of lumbar disc herniation
\end{abstract}

Keywords: Intervertebral disc displacement; Diskectomy; Lumbar vertebrae; Physical examination; Spinal diseases.

\section{RESUMEN}

Objetivo: Investigar la correlación entre las maniobras de estiramiento del nervio ciático, como la Prueba de Lasègue (PL) y la Prueba de Elevación de la Pierna Extendida (PEPE), con los resultados quirúrgicos en pacientes con ciática. Métodos: 178 pacientes con hernia discal lumbar fueron examinados previamente por el autor mediante la PL y la PEPE. Los resultados quirúrgicos fueron registrados y comparados con los hallazgos en el examen clínico. Resultados: De los pacientes estudiados, 162 (91\%) fueron positivos en la PL, mientras que 118 (66,2\%) presentaron PEPE positiva. Conclusión: La PL fue la maniobra de diagnóstico preoperatorio más sensible en correlación con hernia discal lumbar.

Descriptores: Desplazamiento del disco intervertebral; Discectomía; Vértebras lumbares; Examen físico; Enfermedades de la columna vertebral.

\section{INTRODUÇÃO}

A medula espinhal, a cauda equina e as raízes nervosas são vulneráveis a muitas desordens potencialmente causadoras de ciatalgia. A patologia mais comumente associada à ciatalgia é a hérnia de disco intervertebral. O primeiro indício de irritação de raiz nervosa é a dor ciática, relatada como dor lancinante ou em queimação que se irradia inferiormente pelas faces posteriores ou laterais da perna, frequentemente associada com parestesia ${ }^{1,2}$. Muitos autores previamente descreveram o mecanismo patofisiológico da dor ciática e dos déficits neurológicos causados pela hérnia discal lombar. A isquemia é causada pela compressão mecânica da raiz nervosa lombar ${ }^{3,4}$, ou por vários fatores químicos do núcleo pulposo causando desmielinização da raiz nervosa ${ }^{5,6}$.

Inúmeras manobras para estirar o nervo ciático e, assim, reproduzir sua dor foram descritas na literatura ao longo dos anos e sua reprodutibilidade, valor preditivo e correlação com achados cirúrgicos têm sido estudadas desde então ${ }^{7}$. O Teste de Elevação da Perna Estendida (TEPE) (Straight Leg Lift) descrita por Lazar Lazarevic, em 1980, consiste na elevação do membro inferior com o joelho estendido, tendo como objetivo demonstrar a tensão do nervo ciático (Figura 1).

\footnotetext{
1 - Médico residente do Serviço de Ortopedia eTraumatologia do Hospital São Lucas da PUCRS - Porto Alegre, RS, Brasil.

2 - Professor Doutor. Membro do Grupo de coluna do Hospital São Lucas da PUCRS - Porto Alegre, RS, Brasil.

3 - Mestrando. Membro do Grupo de Coluna do Hospital São Lucas da PUCRS - Porto Alegre, RS, Brasil.

4 - Professor da Faculdade de Medicina da PUCRS, Mestre em Neurocirurgia, Membro do Grupo de Coluna do Hospital São Lucas da PUCRS - Porto Alegre, RS, Brasil.

5 - Professor Doutor da Faculdade de Medicina da PUCRS. Membro do Grupo de Coluna do Hospital São Lucas da PUCRS - Porto Alegre, RS, Brasil.
} 
O teste de elevação da perna retificada é o mais significante teste para diagnóstico de hérnia discal lombar e avaliação da progressão desta condição ${ }^{8}$. O Teste de Lasègue (TL), publicado em 1881 por Forst, discípulo de Charles Lasègue, é composto de duas etapas: primeiramente, realiza-se o TEPE, como apresentada por Lazarevic, seguida, então, pela flexão do quadril com o joelho fletido (Figura 2), diferenciando a dor ciática da dor da articulação coxo-femoral. Estas duas manobras, juntamente com a Elevação Estendida da Perna Contralateral (Cross-Leg ou well-Legg Sign) publicada por Fajerszajn, são algumas das mais conhecidas e utilizadas no nosso meio ${ }^{9,10}$.

O objetivo do presente trabalho foi comparar os achados do exame físico, através do TL e do TEPE com os achados intra-operatórios: tipo, nível topográfico e a localização da hérnia em relação à raiz nervosa.

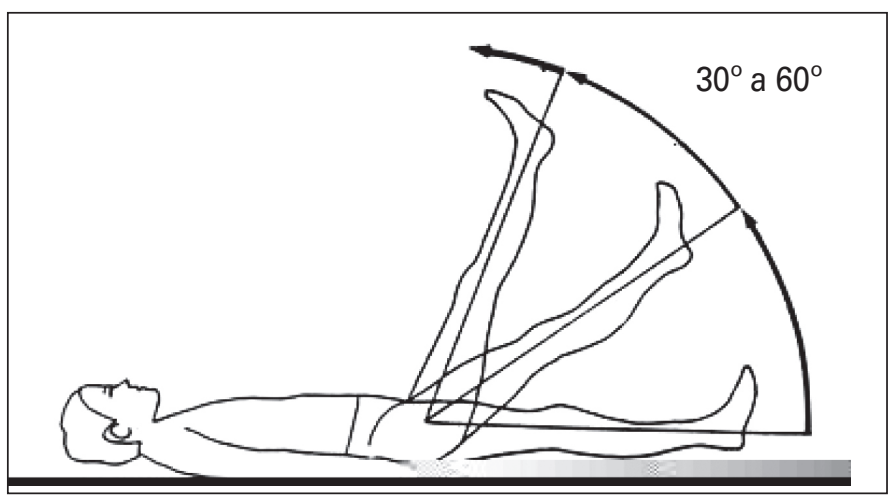

Figura 1. Teste de Elevação da Perna Estendida.

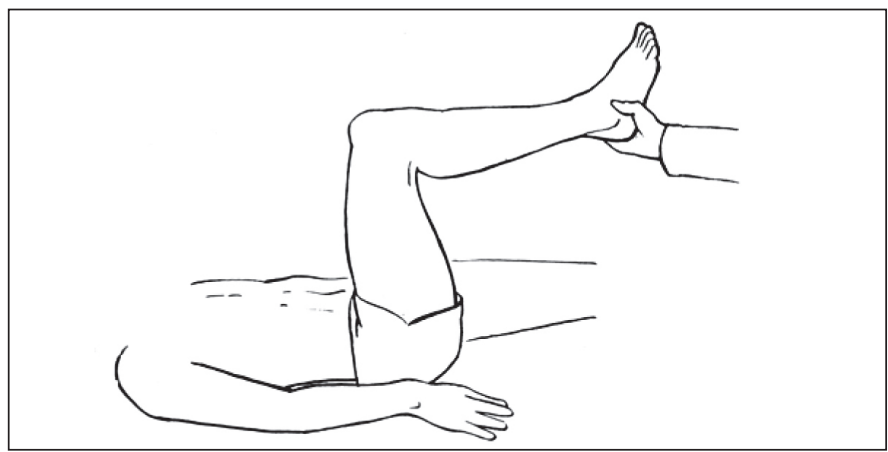

Figura 2. Manobra de Flexão do Quadril, etapa complementar do Teste de Lasègue descrito por Forst.

\section{MATERIAIS E MÉTODOS}

Foi realizada uma análise retrospectiva de 186 pacientes submetidos a tratamento cirúrgico de hérnia de disco lombar no Serviço de Ortopedia e Traumatologia do Hospital São Lucas da PUCRS de Porto Alegre, no período de janeiro de 2006 a julho de 2010. Cento e setenta e oito pacientes apresentavam como queixa principal ciatalgia. Três pacientes apresentavam lombalgia e outros três, claudicação neurogênica. Dois deles apresentavam, apenas, déficit motor do tríceps sural, tendo sido encaminhados ao serviço e submetidos à cirurgia por dano radicular importante. Estes últimos pacientes foram excluídos do estudo, o que limitou a análise aos casos de ciatalgia pura (178 pacientes). O diagnostico de hérnia discal, o tipo e sua localização foram estabelecidos através da realização de um ou mais dos seguintes exames por imagem: tomografia computadorizada em 43 pacientes e ressonância nuclear magnética em 171 pacientes. Foi usada a classificação seguindo as orientações incluídas nas diretrizes de hérnia de disco lombar no adulto jovem ${ }^{11}$ O diagnóstico foi confirmado pela cirurgia em todos os casos.

No exame físico do grupo de pacientes estudado a avaliação do estiramento do nervo ciático foi realizada através do TL e do TEPE. Durante o exame, as seguintes medidas foram tomadas: os pacientes eram posicionados em decúbito dorsal, a perna era elevada com o joelho estendido (TEPE) e em seguida era realizada a flexão do quadril com o joelho fletido, completando o TL descrito por Frost. Nosso TL incluiu, ainda, a manobra de dorsiflexão do pé, descrita por Lazarevic, e utilizada por inúmeros autores ${ }^{9}$. (Figura 3).

Os testes foram considerados positivos quando reproduziam dor entre $30^{\circ}$ e $60^{\circ}$ de elevação ${ }^{12}$ tanto nas costas, quanto no membro inferior. Não foram utilizados equipamentos especiais para a medição do ângulo de flexão do quadril uma vez que todos os pacientes foram examinados pela mesma pessoa sendo a estimativa visual confiável uma vez que não houve avaliação inter-observadores $^{1,13}$. O examinador e o cirurgião foram pessoas diferentes.

Baseado nos achados transoperatórios e nos exames de imagem as hérnias foram classificadas conforme o seu grau em: protrusas, extrusas e seqüestradas; quanto a sua localização em relação à raiz nervosa, em: subradiculares, laterais à raiz, médio radiculares e seqüestradas e de acordo com seu nível topográfico. Os dados foram arquivados no programa Excel e avaliados pelo programa BioEstat 5.0. Foi avaliada a sensibilidade dos testes.

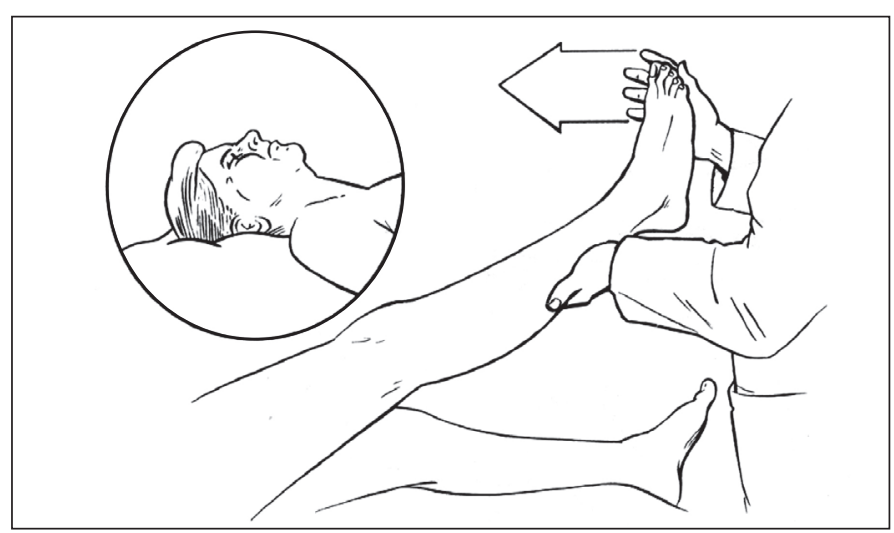

Figura 3. Manobra de dorsiflexão do Pé segundo Lazarevic.

\section{RESULTADOS}

A classificação dos pacientes quanto ao tipo de hérnia diagnosticada através de exames de imagem está discriminada na Tabela 1.

Dos 178 pacientes estudados, 162 (91\%) apresentaram TL positivo enquanto 117 (65,7\%) apresentaram o TEPE positivo. Quanto ao nível topográfico da hérnia encontramos três casos no nível L2-L3, cinco casos em L3-L4, 66 casos em L4-L5 e 104 casos no nível L5-S1. A correlação entre o exame físico e o nível topográfico está descrita na Tabela 2.

$\mathrm{O}$ TL provocou dor adicional no subgrupo de pacientes que apresentou o TEPE positivo $(n=85)$. As correlações do exame físico com a localização trans-operatória da hérnia em relação à raiz nervosa encontra-se na Tabela 3.

Na série estudada os diagnósticos dos exames por imagem foram compatíveis com os achados do trans-operatório no que se refere à posição e ao tipo de hérnia.

Tabela 1. Classificação dos pacientes quanto ao tipo de hérnia diagnosticada através dos exames de imagem.

\begin{tabular}{c|c|c|c|c}
\hline Colunas 1 & Protusão & Extrusão & Sequestrada & Total \\
\hline & $\mathrm{N}=26(14,6 \%)$ & $\mathrm{N}=125(70,2 \%)$ & $\mathrm{N}=27(15,1 \%)$ & 178 \\
\hline Lasègue & $24(92,3 \%)$ & $123(98,4 \%)$ & $15(55,5 \%)$ & 162 \\
\hline $\begin{array}{c}\text { Elevação da perna } \\
\text { estendida }\end{array}$ & $13(50 \%)$ & $95(76 \%)$ & $9(33,3 \%)$ & 117 \\
\hline
\end{tabular}

Tabela 2. Exame físico e nível topográfico da hérnia.

\begin{tabular}{c|c|c|c|c|c}
\hline Colunas 1 & L2-L3 & L3-L4 & L4-L5 & L5-S1 & Total \\
\hline & $\mathrm{N}=3(1,6 \%)$ & $\mathrm{N}=5(2,8 \%)$ & $\mathrm{N}=66(37 \%)$ & $\mathrm{N}=104(58,4 \%)$ & $17(100 \%)$ \\
\hline Lasègue & 3 & 4 & 60 & 99 & $162(91 \%)$ \\
\hline Sensibilidade & $100 \%$ & $80 \%$ & $90,90 \%$ & $95,10 \%$ & \\
\hline $\begin{array}{c}\text { Elevação da perna } \\
\text { estendida }\end{array}$ & 2 & 3 & 64 & 57 & $118(66,2 \%)$ \\
\hline Sensibilidade & $66,60 \%$ & $60 \%$ & $96,90 \%$ & $54,80 \%$ & \\
\hline
\end{tabular}


Tabela 3. Exame físico e localização trans-operatória.

\begin{tabular}{c|c|c|c|c|c}
\hline Colunas 1 & $\begin{array}{c}\text { Sub Radicular } \\
\mathbf{N = 5 3 ( 2 9 , 7 \% )}\end{array}$ & $\begin{array}{c}\text { Lateral à Raiz } \\
\mathbf{N = 6 4}(\mathbf{3 5 , 9 \% )})\end{array}$ & $\begin{array}{c}\text { Médio Radicular } \\
\mathbf{N = 3 4 ( 1 9 , 1 \% )}\end{array}$ & $\begin{array}{c}\text { Sequestrada } \\
\mathbf{N = 2 7 ( 1 5 , 1 \% )}\end{array}$ & Total 178 \\
\hline Lasègue & 50 & 63 & 34 & 15 & 162 \\
\hline Sensibilidade & $94,30 \%$ & $98,40 \%$ & $100 \%$ & $55,50 \%$ & \\
\hline $\begin{array}{c}\text { Elevação } \\
\text { da perna } \\
\text { estendida }\end{array}$ & 33 & 46 & 29 & 9 & 117 \\
\hline Sensibilidade & $62,20 \%$ & $71,80 \%$ & $85,20 \%$ & $33,30 \%$ & \\
\hline
\end{tabular}

\section{DISCUSSÃO}

É importante salientar, em primeiro lugar, a dificuldade encontrada para comparar nossos resultados com muitos trabalhos pelo fato de os autores raramente descreverem o método utilizado para reproduzir a dor ciática. Além disso, apesar de o TL ser um dos epônimos mais comumente utilizados na Prática médica, frequentemente é confundido com o TEPE ${ }^{14}$.

Em 1988, Kosteljanetz et al. ${ }^{12}$ estudaram 55 pacientes com dor ciática unilateral com o objetivo de avaliar a variação do resultado do TL entre observadores e também avaliar a correlação do teste com os achados cirúrgicos. Neste estudo os autores consideraram teste positivo quando este produzia dor na perna ipsilateral (Lasègue típico) ou apenas nas costas (Lasègue atípico) quando era realizado o TEPE. Dos 55 pacientes, 52(94,5\%) foram submetidos à cirurgia com base nos achados clínicos e de exames por imagem e destes, 45(81,8\%) tiveram diagnóstico cirúrgico de hérnia de disco. Considerando apenas os pacientes com teste típico os autores encontraram uma sensibilidade de $88 \%$ para o TL e quando acrescentados aqueles com teste atípico a sensibilidade chegou a $95,5 \%$. Em nosso trabalho encontramos uma sensibilidade de 91\% para o TL.

Jönsson e Strönquvist ${ }^{15}$ realizaram um estudo prospectivo para determinar a frequência dos sinais e sintomas mais comuns em 300 pacientes com síndromes compressiva de raiz nervosa lombar, dos quais 100 apresentavam hérnia de disco lombar. O TEPE foi positivo em $88 \%$ dos pacientes com hérnia e em 98\%, se consideradas apenas as hérnias completas. Dos 178 pacientes estudados em nosso trabalho 118 (66,2\%) apresentaram o TEPE positivo.

Neste mesmo ano, Supik e Broom ${ }^{9}$ estudaram 50 pacientes com diagnóstico clínico e radiológico de hérnia de disco com o objetivo de correlacionar as manobras de estiramento do nervo ciático com a localização anatômica das hérnias. Os autores compararam quatro tipos de manobras, incluindo as duas utilizadas em nosso estudo: o TL (utilizando também a dorsiflexão do pé) e o TEPE. Encontraram uma sensibilidade de 96\% para o TEPE e de $71 \%$ para o TL. Quando as manobras foram comparadas com os níveis topográficos das hérnias, o TEPE também foi mais acurado. O estudo foi incapaz de demonstrar correlação entre as manobra e a localização da hérnia em relação à raiz nervosa.

\section{REFERÊNCIAS}

1. Deyo RA, Rainville J, Kent DL. What can the history and physical examination tell us about low back pain? JAMA. 1992;268(6):760-5

2. Iglesias-Casarrubios P, Alday-Anzola R, Ruíz-López P, Gómez-López P, Cruz-Bértolo J, Lobato RD. [Lasegue's test as prognostic factor for patients undergoing lumbar disc surgery]. Neurocirugia (Astur). 2004:15(2):138-43.

3. Olmarker K, Rydevik B, Holm S, Bagge U. Effects of experimental graded compression on blood flow in spinal nerve roots. A vital microscopic study on the porcine cauda equina. J Orthop Res. 1989;7(6):817-23.

4. Olmaker K. The experimental basis of sciatica. J Orthop Sci. 1996;1:230-42.

5. Hida S, Naito M, Kubo M. Intraoperative measurements of nerve root blood flow during discectomy for lumbar disc herniation. Spine (Phila Pa 1976). 2003;28(1):85-90

6. Rydevik B, Brown MD, Lundborg G. Pathoanatomy and pathophysiology of nerve root compression. Spine (Phila Pa 1976). 1984;9(1):7-15

7. Albeck MJ. A critical assessment of clinical diagnosis of disc herniation in patients with monoradicular sciatica. Acta Neurochir (Wien). 1996:138(1):40-4

8. Takamori Y, Arimizu J, Izaki T, Naito M, Kobayashi T. Combined measurement of nerve root blood flow and electrophysiological values: intraoperative straight-leg-raising test for lumbar disc herniation. Spine (Phila Pa 1976). 2011;36(1):57-62.

9. Supik LF, Broom MJ. Sciatic tension signs and lumbar disc herniation. Spine (Phila Pa 1976). 1994;19(9):1066-9

10. Dyck P. Lumbar nerve root: the enigmatic eponyms. Spine (Phila Pa 1976). 1984;9(1):3-6.

11. Façanha Filho FAM, Defino H, Gonzaga MC, Zylbersztejn S, Meves R, Canto FT, et al
Em 1996, Vucetic e Svensson ${ }^{14}$ estudaram a capacidade de vários sinais clínicos, entre eles o Teste de Lasègue, se correlacionarem com os achados cirúrgicos, em 163 pacientes com hérnia de disco, incluindo o grau da hérnia (completa, incompleta, protrusa ou com disco normal), o nível topográfico e a localização da hérnia em relação à raiz nervosa (sub radicular, lateral à raiz, médio radicular e seqüestrada). O TL neste estudo foi realizado conforme descrito por Forst e foi considerado positivo apenas se fosse reproduzida dor no membro inferior ipsilateral. O TL apresentou uma sensibilidade de aproximadamente $86 \%$ mas os autores também não conseguiram demonstrar correlação entre os achados clínicos e cirúrgicos. É sabido na literatura que este teste encontra uma baixa especificidade. ${ }^{(16)}$ No entanto, o TEPE correlaciona-se muito bem com as forças objetivas medidas, demonstrando a confiabilidade do teste ${ }^{17}$. Melhor desempenho pode ser obtido com a combinação dos testes diagnósticos, incluindo informações tanto da anamnese quanto do exame físico ${ }^{18}$.

Na revisão dos dados obtidos no presente trabalho encontrados na nossa casuística identificamos alto índice de positividade dos testes de estiramento do nervo ciático no grupo estudado e este dado pode ter relação com o fato destes pacientes já terem sido encaminhados ao nosso serviço para realização de tratamento cirúrgico por lombociatalgia intensa e incapacitante e/ou sinais de lesão radicular grave. Outro motivo para o alto índice de positividade é o fato de a maioria dos nossos pacientes apresentarem extrusão $(70,2 \%)$ com importante compressão radicular.

Encontramos maior sensibilidade do TL $(95,1 \%)$ em comparação com o TEPE(54,8\%) nos 104 pacientes com hérnia no nível L5-S1, mas estes dados não permitem concluir se há realmente maior correlação dos testes com o nível topográfico, pois o número de pacientes portadores de Hérnia Discal em outros níveis foi menor . O trabalho também não nos permitiu correlacionar as manobras de estiramento do nervo ciático com a localização da hérnia em relação à raiz nervosa.

Paradoxalmente observamos grande positividade nos TL e TEPE nas hérnias dos níveis L2-L3 e L3-L4. Esta positividade pode estar relacionada ao volume e posição do material discal herniado. Estes pacientes apresentavam volumosas hérnias extrusas e seqüestradas que comprometiam de maneira significativa o canal medular e as raízes da cauda equina.

\section{CONCLUSÃO}

O TL e o TEPE apresentaram alto índice de positividade no grupo de pacientes avaliados, portadores de hérnia discal lombar. Encontramos maior sensibilidade do TL em comparação com o TEPE nos pacientes com hérnia no nível L5-S1, 95,1\% e 54,8\% respectivamente, mas devido ao menor número de portadores de hérnia discal em outros níveis, não podemos concluir se há maior correlação com o nível topográfico. O presente estudo nos mostra, ainda, a importância dos testes de estiramento do nervo ciático no exame físico dos pacientes com suspeita de radiculopatia por hérnia discal sugerindo investigação adicional por imagem neste subgrupo de pacientes.

Hérnia de disco lombar no adulto jovem. Projeto Diretrizes. São Paulo: Associação Médica Brasileira e Conselho Federal de Medicina; 2007.

12. Kosteljanetz M, Bang F, Schmidt-Olsen S. The clinical significance of straight-leg raising (Lasègue's sign) in the diagnosis of prolapsed lumbar disc. Interobserver variation and correlation with surgical finding. Spine (Phila Pa 1976). 1988:13(4):393-5.

13. McCombe PF, Fairbank JC, Cockersole BC, Pynsent PB. 1989 Volvo Award in clinical sciences. Reproducibility of physical signs in low-back pain. Spine (Phila Pa 1976). 1989;14(9):908-18.

14. Vucetic N, Svensson O. Physical signs in lumbar disc hernia. Clin Orthop Relat Res. 1996:(333):192-201.

15. Jönsson B, Strömqvist B. Symptoms and signs in degeneration of the lumbar spine A prospective, consecutive study of 300 operated patients. J Bone Joint Surg Br. 1993;75(3):381-5.

16. Devillé $W L$, van der Windt DA, Dzaferagic A, Bezemer PD, Bouter LM. The test of Lasègue: systematic review of the accuracy in diagnosing herniated discs. Spine (Phila Pa 1976). 2000;25(9):1140-7.

17. Mens JM, Pool-Goudzwaard A, Beekmans RE, Tijhuis MT. Relation between subjective and objective scores on the active straight leg raising test. Spine (Phila Pa 1976). 2010:35(3):336-9.

18. van der Windt DA, Simons E, Riphagen II, Ammendolia C, Verhagen AP, Laslett M, et al. Physical examination for lumbar radiculopathy due to disc herniation in patients with low-back pain. Cochrane Database Syst Rev. 2010;(2):CD007431. 